\title{
A Replicator Dynamics Model of Traditional Manufacturing Clusters Low-carbon Evolution
}

\author{
Hongjuan Zhang ${ }^{1,2}$ \\ ${ }^{1}$ Management School, Wuhan University of Science and Technology, Wuhan, Hubei, 430081, China \\ ${ }^{2}$ Center for Industrial Policy and Management Research, Wuhan, Hubei, 430081, China \\ jhz75@126.com
}

\begin{abstract}
Keywords:Manufacturing Cluster,Replicator Dynamics Model,Cooperative Evolution, Evolutionarily Stable Strategy

Abstract.The low-carbondevelopmentof traditional manufacturing clustershas becomea hot issuein thecluster field. The cooperative evolution is animportant way of the low-carbon evolutionof traditional manufacturing clusters, and it is also a dynamical, complex and repeatedgame process. This paper builds a replicator dynamics model of traditional manufacturing clusterlow-carbon evolution. Based on evolutionary game theory, the paper analyzes the low-carbon evolutionary paths and evolutionarily stable strategy of traditional manufacturing clusterunder different initial states of cluster system. The analytic results show that, the result of low-carbon evolution depends on the initial conditions of cluster system. The paper studies the mechanism of cluster enterprises changing their low-carbon behavior strategy according to the external environmental changes, and has a good practical value and significance.
\end{abstract}

\section{Introduction}

The low-carbon developmentof traditional manufacturing clustershas becomea hot issue in theclusterfield, and it is also an important part in the development of low carbon economy[1].

The key influence factor to low-carbon development of traditional manufacturing clusteris R\&D strength. Cooperative low-carbon strategy as a major strategy of cluster low-carbon developmentstrategies has many advantages, such as small risk, low cost and advanced technology[2].In order to share resources such as capital, information and technology, cluster enterprises would prefer to choose adopting cooperative strategyto obtain the advantage of low carbon development[2].

The cooperative low-carbon evolution of traditional manufacturing clusters system is a long and complex process. The low-carbon evolutionary path and evolutionarily stable strategy of cluster system are all not uncertain[3].

Replicator dynamics model can describethe behaviorstrategy of the players in the process of game well, and it canpredict the evolutionary path and evolutionarily stable strategy of the gamewell [4].So based onreplicator dynamics model of monomorphic population, the paper can describe and predict the cooperative low-carbon evolution of traditional manufacturing clustervery well.Therefore, thispaper would use replicator dynamics model to study the low-carbon evolutionary mechanism of traditional industrial cluster, and to analyze the changes of cluster enterprise low-carbon behavior strategy according to the external environmental changes.

\section{Modeling the Replicator Dynamics Model of Cluster Low-carbon Evolution}

\section{Basic Replicator Dynamics Modelof Monomorphic Population}

In the monomorphic population symmetric game, assuming that $N$ indicates the total numberof agents of populaiton, $S^{K}=\left\{s_{1}, s_{2}, \ldots, s_{k}\right\}$ indicates the pure strategy sets of game agents. And suppose that every agenthave to adopt a pure strategy at any time. $n_{i}(t)$ indicates the number of agents adopting pure strategy $i$ at time $t . x_{i}$ indicates the proportion of agents adopting pure strategyi at timet, then $x_{i}$ can be expressed as follow $[4,5]$ :

$$
x_{i}=n_{i}(t) / N
$$


Assuming that the expected revenueof agent who adopting pure strategyi can be expressed as $f\left(s_{i}, x\right)$, then the average expected revenueof population can be expressed as follow:

$\bar{f}(x, x)=\sum x_{i}\left(s_{i}, x\right)$

So the basic replicator dynamics modelof monomorphic populationcan be expressed as follow:

$$
d x_{i}(t) / d t=\left[f\left(s_{i}, x\right)-\bar{f}(x, x)\right] x_{i}
$$

\section{Modeling the Replicator Dynamics Model of Traditional Manufacturing Cluster Low-carbon Evolution}

(1)Suppose that the traditional manufacturing clusteris composed of a monomorphic population of enterprises, and the totalnumber of cluster enterprises is $N(N \geqslant 2)$.

(2)Suppose that when the manufacturing clusterenterprises game with each other, they have the same pure Strategy Sets, that is $S=\left\{S_{1}, S_{2}\right\}$. $S_{1}$ is "Cooperative Low-carbonStrategy", $S_{2}$ is "Independent Low-carbonResearch Strategy". And suppose that every manufacturing cluster enterprise have to choose adopting a pure strategyat any time.

(3)Suppose that the proportion of traditional manufacturing cluster enterprises adopts low-carbon strategy $S_{1}$ is $x$, and the proportion adopts low-carbonstrategy $S_{2}$ is $1-x(0 \leqslant \mathrm{x} \leqslant 1)$.

(4)Assuming that all thetraditional manufacturingcluster enterprises in the game distribute low-carbon cooperative benefit evenly, have to pay the same low-carbon cooperative cost, and have the same low-carbon speculative revenue.

Table 1 shows the Game Payoff Matrix of a monomorphic population of traditional manufacturing cluster enterprise.

As shown in Table 1,

$u$ is thetraditional manufacturing cluster enterpriseprofit whenit isadoptinglow-carbon strategy $S_{2}$ in the game;

$c$ is thetraditional manufacturing cluster enterprisecooperative cost when it isadoptinglow-carbon strategy $S_{1}$;

$k$ is thetraditional manufacturing cluster enterprise speculative revenue when it isadoptinglow-carbon strategy $S_{2}$, while the other side game participantis adopting low-carbon strategy $S_{1}$;

$h$ is the traditional manufacturing cluster enterprise coefficient of profitability;

$h-c$ is thetraditional manufacturing cluster enterprisecoefficient of cooperative profitability, when all the game participants are adopting low-carbon strategy $S_{1}$.

Table1Game Payoff Matrixof manufacturingclusterenterprise 1

\begin{tabular}{|c|c|c|}
\hline & $S_{1}$ & $S_{2}$ \\
\hline$S_{1}$ & $u+h-c, u+h-c$ & $u-c, u+k$ \\
\hline$S_{2}$ & $u+k, u-c$ & $u, u$ \\
\hline
\end{tabular}

In order to simplify the calculation, the paper sets $u=0$. Thisset would not affect the results of the game analysis. Then Table 1 is simplified to Table 2.According to the actual game situations of traditional manufacturing clusterenterprises, the parameters are set as follows: $h>c, k>0, c>$ 0 . 
Table 2Game Payoff Matrix of manufacturingclusterenterprise 2

\begin{tabular}{|c|c|c|}
\hline & $S_{1}$ & $S_{2}$ \\
\hline$S_{1}$ & $h-c, h-c$ & $-c, k$ \\
\hline$S_{2}$ & $k,-c$ & 0,0 \\
\hline
\end{tabular}

Whentraditional manufacturing cluster enterprise is adopting low-carbon strategy $S_{1}$, its average expected revenuev $v^{1}$ can be described as Eq. 1:

$v^{1}=(h-c) x+(-c)(1-x)=h x-c(1)$

Whentraditional manufacturing cluster enterprise is adopting low-carbon strategy $S_{2}$, its average expected revenue $v^{2}$ can be described as Eq. 2:

$v^{2}=k x(2)$

Then the Replicator Dynamics Equation of traditional manufacturing cluster enterprise can be described asEq. 3:

$$
F=\frac{d x}{d t}=x\left(v^{1}-v\right)=x(1-x)\left(v^{1}-v^{2}\right)=x(1-x)(h \mathrm{x}-k \mathrm{x}-c)(3)
$$

\section{Analysis of Evolutionarily Stable Strategy of Traditional Manufacturing Cluster Low-carbon Evolution}

In Eq.3, suppose that $F=\frac{d x}{d t}=0$, then $x_{1}^{*}=0, x_{2}^{*}=1, x_{3}^{*}=\frac{c}{h-k}$. It means only when $x^{*}=$ 0,1 or $\frac{c}{h-k}$, the proportion of traditional manufacturing cluster enterprises which are adopting strategy $S_{1}$ is stable.

Based on evolutionary game theory, suppose that $F^{\prime}\left(x^{*}\right)<0$, then $x^{*}$ is evolutionarily stable strategy (ESS)of traditional manufacturing cluster low-carbon evolution. That means the evolutionarily stable strategy of cluster enterprise low-carbon evolution depends entirely on the parameters of the game payoff matrix $(h, k, c)[6]$.

$$
\begin{aligned}
& F^{\prime}\left(x_{1}^{*}\right)=F^{\prime}(0)=-c \\
& F^{\prime}\left(x_{2}^{*}\right)=F^{\prime}(1)=k-(h-c) \\
& F^{\prime}\left(x_{3}^{*}\right)=F^{\prime}\left(\frac{c}{h-k}\right)=\frac{c(h-c-k)}{h-k}
\end{aligned}
$$

Since $h>c, k>0, c>0$, then $F^{\prime}(0)=-c<0$. According to the different values of $h, k, c$, the result of evolutionarily stable strategy of traditional manufacturing cluster can be divided as following situations[6]:

(1)When $h-c>k$ and $h<k$, this situation does not exist.

(2) When $h-c>k$ and $h>k$, it can be conducted that $F^{\prime}\left(x_{1}^{*}\right)<0, F^{\prime}\left(x_{2}^{*}\right)<0, F^{\prime}\left(x_{3}^{*}\right)>0$, therefore $x_{1}^{*}=0$ and $x_{2}^{*}=1$ are bothevolutionarily stable strategy (ESS).

Sincethe final result of low-carbon evolution depends on the initial value of $x$, so when $x \in$ $\left(\begin{array}{ll}0, x_{3}^{*}\end{array}\right)$, through long-term repeated game,traditional manufacturingcluster enterprises finally would adopt independent low-carbon researchstrategy; when $x \in\left(x_{3}^{*}, 1\right)$, through long-term repeated game, cluster enterprises finally would adopt cooperative low-carbon strategy.

(3)When0 $<h-c<k$ and $h<k$,

it can be conducted that $F^{\prime}\left(x_{1}^{*}\right)<0, F^{\prime}\left(x_{2}^{*}\right)>0, F^{\prime}\left(x_{3}^{*}\right)>0$, therefore, $x_{1}^{*}=0$ is the only evolutionarily stable strategy. It means through long-term repeated game, traditional manufacturing cluster enterprises finally would adopt independent low-carbon research strategy

(4)When $0<-c<k$ and $h>k$,

it means $k<h<c+k$, it can be conducted that $F^{\prime}\left(x_{1}^{*}\right)<0, F^{\prime}\left(x_{2}^{*}\right)>0, F^{\prime}\left(x_{3}^{*}\right)<0$, 
therefore $x_{3}^{*}$ is not evolutionarily stablestrategy, $x_{1}^{*}=0$ is the only evolutionarily stable strategy. It means through long-term repeated game, traditional manufacturing cluster enterprises finally would adopt independent low-carbon research strategy.

According to the actual game situations of traditional manufacturing clusterenterprises, other values ofparametersof game payoff matrixdon't exist.

\section{Conclusions}

The low-carbon cooperative evolution oftraditional manufacturing clusters is a dynamical, complex and repeatedgame process. This paper builds a replicator dynamics model oftraditional manufacturing clusterslow-carbon evolution. Based on evolutionary game theory, the paper analyzes the low-carbon evolutionary paths and evolutionarily stable strategy of traditional manufacturing clusterunder different initial states of cluster system. The analysis results show that,the low-carbon evolutionary results of traditional manufacturing clusterdepend on the initial conditions of cluster system.The paper studies the mechanism of traditional manufacturing cluster enterprises changing their low-carbon behavior strategy according to the external environmental changes, and has a good practical value and significance.

\section{Acknowledgements}

This work was financially supported byMOE(Ministry of Education in China) Project of Humanities and Social Science (No.15YJCZH221),Foundationof Center for Industrial Policy and Management Research(No.CY20150211), and Youth Project ofWuhanUniversity of Science and Technology(No.2015XZ021).

\section{References}

[1]G.Y Zhuang: Word Environment, Vol. 2(2008):34-36

[2] R.G Fan, H.J Zhang:Applied Mechanics and Materials, Vols.448 (2014)

[3]Friedman D:Theory Journal of Evolutionary Economics, Vol. 8(1998):15-43.

[4] Peter D. Taylor:Journal of Applied Probability,Vol.16 (1979):76-83

[5]S.YXie,Economic Game Theory, Shanghai: Fudan University Press, (2002)(In Chinese)

[6]Selten R:Journal of Theoretical Biology, Vol. 84 (1980):93-101 